\title{
English Proverbs into Arabic through Machine Translation
}

\author{
Mohammad H. Al-khresheh ${ }^{1 *}$, Shahab A. Almaaytah ${ }^{2}$ \\ ${ }^{1}$ Department of English language, Northern Border University, Saudi Arabia \\ ${ }^{2}$ King Faisal University, Saudi Arabia
}

Corresponding Author: Mohammad H. Al-khresheh, E-mail: mohd_khresheh@yahoo.com

\begin{tabular}{l} 
ARTICLE INFO \\
\hline Article history \\
Received: March 14, 2018 \\
Accepted: May 15, 2018 \\
Published: September 01,2018 \\
Volume: 7 Issue: 5 \\
Advance access: July 2018 \\
\hline
\end{tabular}

Conflicts of interest: None Funding: None

\begin{abstract}
Proverbs are sentences used by people to convey wisdom, truth or morals that have been handed down for generations. Translating them from one language into another through machine translation has been a matter of interest because of their importance in keeping the religious, cultural and historical aspects. The accuracy of machine translation systems has become one of the most important issues nowadays as the demand for online translation is increasing. Therefore, the current study aimed at investigating the linguistic obstacles that machine translation might face in carrying out the same translation from English proverbs into Arabic. It also attempted to confirm the importance of human intervention in solving the issue of accuracy. To achieve these objectives, a group of English proverbs were randomly selected, translated into Arabic by 'Google translate' and analysed qualitatively by the researchers. On the one hand, the findings revealed that 'Google translate' faced some linguistic obstacles in carrying out the same meaning of English proverbs into Arabic. Lexically, words that have multiple meanings present countless challenges and difficulties to online translation. On the other hand, the different linguistic structures between English and Arabic make the process of translation sometimes a bit challenging as both languages belong to unrelated families. Simply put, machine translation is expected to encounter various problems in providing meaningful logical translation. A detailed discussion is provided and recommendations for further research are given based on the analysis.
\end{abstract}

Key words: Arabic, English, Machine Translation, Proverbs

\section{INTRODUCTION}

English is one of the most widely spoken languages in the world. It is the official language of more than 53 countries. It is the language of media, science, commerce, and industry (Al-khresheh, 2013). In spite of this importance, not everyone can speak it for multiple reasons. Therefore, understanding the surrounding world forces us to understand this commonly-spoken language. In this regard, translation plays an essential role in facilitating the process of understating different languages. Khalifa (2015) pointed out that "the needs for translation are increasing nowadays due to the continuous development of science, culture, and technology. However, the process of translation is faced by many linguistic problems including grammar, context, culture, etc" (p. 22).

Given this, translation is "the process of transferring a written text from source language to target language" (Hatim \& Munday, 2004, p. 6). This can help us produce natural equivalent of the source language (SL) text in the target language (TL). Translation can be defined as a process that works on linguistic signs, consisting of both the linguistic as well as cultural aspects of the given text, and such a process can take place between two different languages or within the same language (Khalifa, 2015). Among the various definitions of translation, the most prominent one is given by Newmark (1988) who defined translation as "rendering the meaning of a text into another language in the way that the author intended the text" (p.5).

Translation of English texts into Arabic using machine translation (MT) has not been successful in giving the best outputs in some situations as these two languages are not from the same family. Denkowski (2015) asserted that to break the language barriers globally there is a pressing need for precision in conveying messages that are of great importance politically especially by international organizations and governments. He also added that to be precise, a human intervention is required in situations where communities come from different language backgrounds. To have a successful human intervention, the translator should be very much familiar with linguistic and even non-linguistic features of both languages as well as with SL culture and TL culture (Thalji, 2015). This might be a bit difficult for MTs; 
hence, it is expected that MT to face different problems in providing a meaningful translation.

With this in mind, the study aimed at investigating the problems of translating English into Arabic with a main focus on proverbs. A proverb cannot be only translated by looking up the literal meaning of its words in a dictionary. Literal translation of proverbs cannot give the accurate and real meaning. Translating proverbs has always been a matter of difficulty due to their various cultural aspects. According to Ghazala (1995), proverbs are "special, fixed, unchanged phrases which have special, fixed, unchanged meanings" (p. 138). They are multi-word expressions. Anastasiou (2010) confirmed that it is not easy to translate most of the multiword expressions, though a few of them can be translated using MT by keeping them as a separate database. These multi-word expressions including proverbs cannot always be translated by MT. As stated earlier, rendering proverbs into another language must be treated precisely, taking into consideration the religious, cultural and historical aspects in order not to distort the meaning of proverbs.

A lot of attention to and interest in, natural language processing has been developed in recent times. Readers can be made to feel emotional by understanding the objective of a text that goes beyond the literal meaning of the text which is the connotative meaning that they understand while comprehending a text (Feng et al., 2013). Taking this into account, translation requires a deep and mutual understanding of both grammar and culture. As mentioned previously, translators should not only be familiar with the TL structures but also the culture of the society who speak the TL. Translating proverbs cannot be envisaged by the literal definitions of the words they contain. Many researchers reveal that proverbs are one of the trickiest items to translate and machine translation might not completely solve (Khalifa, 2015; Dorr, 1994; Resnik and Yarowsky,1999; Carpuat, 2013 \& 2015).

Although translation technology is continuously improving, the level of accuracy and quality is still very low (Madsen, 2009; Zhengl, 2015). A human intervention is highly needed to ensure the uppermost accuracy. Humans can cautiously understand context in order to capture the identical meaning, rather than merely translating individual words. Therefore, the focus of the study is limited to the linguistic problems that 'Google translate' (GT) might face in dealing with the input of SL and providing the output of TL. Having grammatically and lexically well-formed translation can only be obtained by analysing such linguistic problems. Particularly, the current study has two main objectives: (1) to investigate the linguistic obstacles that GT might face in carrying out the same translation from English proverbs into Arabic, and (2) to confirm the importance of human intervention in solving the issue of accuracy.

Given the above research objectives, the current study does not only gain its significance and rationality from the importance of MT in translating English proverbs into Arabic, but also from the fact that the present study could be differentiated from previous studies that are carried out in this field and the recommendations which could be derived from the research findings. The study is also significant because the translation of proverbs through MT has not been given enough attention by researchers. Knowing about linguistic obstacles encountered by MT offers a window into the human mind and helps us improve the level of accuracy, particularly when dealing with proverbs which are culturally and religiously used by people to convey wisdom and morals. Providing well-formed and meaningful translation remains one of the most important things in the field of translation. People should also understand the fact that online MT does not always provide an accurate translation.

\section{LITERATURE REVIEW}

As the present study sheds light on the problem of translating English proverbs into Arabic, it is important to affirm that proverbs exist in all languages, and depending on the culture the importance of proverbs may vary (Ahmed, 2005; \& Dabaghi, et. al., 2010). Alshammari (2015) stated that proverbs are used to express cultural wisdom. They are present in all languages to convey certain messages. In the same vein, Samover et al. (2009) confirmed that proverbs carry out particular messages for the sake of expressing cultural values and beliefs. According to Meider (1985), a proverb is "a short, generally known sentence of the folk which contains wisdom, truth, morals, and traditional views in a metaphorical, fixed and memorizable form and which is handed down from generation to generation" (p.119). Another definition of proverbs by Mollanazar (2001) is that a proverb is "a unit of meaning in a specific context through which the speaker and hearer arrive at the same meaning" (p.53). Proverbs are still used as a powerful way to communicate in the modern society though in some parts of the society the frequency of their usage may vary. According to Alshammari (2015), this powerful way of communication has two main functions, literary and practical. The former one means that proverbs can be used in prose, poetry, and songs whereas the latter one indicates that proverbs have certain properties that are practical for everyday purposes. They;

are also used for treatment of socio-psychological problems such as substance abuse, psychotherapy, tests of mental status, as a way of teaching children to think more abstractly, as an imaginary mnemonic by the elderly, as a means of assessing workers' attitudes about work and life, and even as tests of a defendant's competency to stand trial. (Honeck, 1997, pp.26-29).

The connotative meaning of the proverbs can be comprehended based on the situation (Mieder, 2004). Consequently, the reader of the source text analyses the proverb, interprets it based on the situation, and formulates the proverb once again according to the situation or context on hand. Both English and Arabic languages have some proverbs that have similar connotation (Ilyas, 2013).

Ilyas (2013) pointed out that "connotation implies the associations that a word may bring to the hearer's mind according to his cognition and experience that are additional to its literal or dictionary meaning"(p. 249). Similarly, Hatim (1997) defined connotation as the "additional meanings that a lexical item acquires beyond its primary, referential meaning" (p. 228). The emotional effect that the words have on 
the listeners as well as the speakers is defined as connotation by Nida (1964). In the same vein, Carpuat (2015) pointed out that connotation is a notion or an emotion evoked by a word which may be positive or negative; anything that people consider as having some worth and can be cherished is the effect of positive connotations while the negative connotations have opposite effects on people. Accordingly, connotation is the negative or positive feeling that a word can evoke in a person who is either the listener or the speaker.

A few research studies have suggested the methods that can be implemented in order to get a better translation of English in Arabic. For example, Sidhu et al.(2010) have suggested mapping of the proverb in the SL and its equivalent in the TL, and this output can be given to the user to get the best results. Hannouna (2004) evaluated three MT systems that translate from English into Arabic cognitively and linguistically. The study focused on certain selected computational and functional criteria of system's performance. The sample consisted of 268 English sentences. Hannouna confirmed that these three MT systems suffer from severe drawbacks particularly related to the syntax and semantics of the targeted translated sentences. The outputs had several deficiencies. Moreover, the Arabic MT output was generally ambiguous and meaningless. The researcher attributed the deficiencies to differences between SL and TL linguistic structures.

Confirming the difficulty of translating English proverbs into other languages, Syzdykov (2013) conducted a study on folk proverbs and sayings considering them an essential part of the culture of human language. His main objective of the study was to interpret English and Kazakh folk proverbs, which can symbolize different features of life, "social experience, outlook, the originality of artistic attitudes and tastes, mental and ethical and aesthetic values" (p, 318). He found that English proverbs are relatively difficult to be translated into other languages. He also stated that proverbs "develop creativity, enrich students' vocabulary, help them to learn the structure of the language, and to develop memory and the emotional expressiveness of speech" (p, 321). Besides, he reported that translating proverbs is not an easy task and further work is highly needed.

Unlike the scope of the current study, Sameer (2016) compared some types of animal English proverbs with Arabic aiming at applying cognitive linguistics to these proverbs along with investigating the type of metonymy and metaphors noticeable in them. To this end, a group of 20 English and Arabic proverbs were collected and analysed. As a result, he found that the selected proverbs, in both languages, share similar semantic molecules and "have the same functions and referring to the same intended meaning" ( $p, 142)$.

Madsen (2009) stated that there are different problems encountered by machine translation. Some problems cannot be solved at all. He also reveals that "high-quality translation done solely by machines is not possible and machine translated texts will continue to be plagued by errors in the future, ranging from eccentric turns of phrase to grave distortions of meaning" (p. 5). Similarly, Zheng1 (2015) reported that regardless of the strong claims of MT's capacity, it still has immense space to improve. He also added that the results obtained from MT were still far from being satisfactory.

Having reviewed some studies on proverbs and MT, it could be revealed that there is a paucity of literature on translating proverbs through MT. Previous studies focused either on proverbs (Sameer, 2016; Syzdykov, 2013) or on problems of MT (Madsen, 2009; Zheng1, 2015). Unlike previous research, the scope of this study is limited to translating uninvestigated English proverbs into Arabic through MT.

\section{METHODOLOGY}

Languages used in the current study are Arabic and English respectively. English is the SL (the language being translated from) whereas Arabic is the TL (the language being translated into). Both languages belong to different families. English belongs to the Indo-European language family whereas Arabic belongs to the Semitic language one (Al-khresheh, 2016a). This difference makes the process of translation a bit complicated and results sometimes in literal translation along with connotative meanings which might be a challenging task. To achieve the main objectives of this study and in order to discover the accuracy of MT, a few English proverbs were randomly selected, translated by GT and analysed qualitatively with reference to the researchers' native language intuition (Arabic) and linguistic knowledge background. As there are many free online translation services, GT was selected and utilised in translating the English proverbs into Arabic as it is the most commonly used one by people who search for a quick translation (Boitet et al. 2009). The analysis was done after providing the equivalent proverbs in Arabic. The nature of mistranslation was presented, explained and discussed.

As mentioned earlier, the focus is on the linguistic problems that GT might face in dealing with the input of SL and giving the output of TL. According to Mollanazar (2001), there are two strategies for translating proverbs: The first strategy is associated with less or more alike form, vocabulary and meaning in case that similar proverbs exist in both SL and TL. The second one suggests that there are numerous proverbs that share the same meaning in the same context with different form and vocabulary. In the following examples, the same deep meaning of both SL and TL can be found, in case that translation is accurate, with different form and vocabulary. However, Al-khresheh (2010) confirms that both languages have differing syntactic word order structures. In case of literal translation, this difference can change the meaning as well. Therefore, MT might is expected to face linguistic obstacles resulting in awkward translation.

\section{ANALYSIS AND DISCUSSION OF FINDINGS}

This section aims at analysing and discussing the translation of six English proverbs into Arabic through MT. As the study mainly investigates the linguistic obstacles that GT might face in carrying out the same translation from English proverbs into Arabic, the aforementioned strategies, by Mollanazar (2001), are followed in terms of form, vocabulary, 
and meaning. Analysing such linguistics problems leads to having lexically and grammatically well-formed translation.

\section{Text 1}

English Proverb: 'A barking dog seldom bites'

Interpretation of this proverb: Do not be afraid of those people who always angrily shout and threaten others, they hardly ever do anything. In other words, actions rarely take place (Speake, 2007, p. 37).

The equivalent proverb in Arabic according to Jabak (2008): ' الكلب النباح لا يعض (p,17).

When this first proverb 'A Barking Dog seldom bites' was tested for translation through GT, the given Arabic translation was 'باح الكلب نادرا ما لدغات' which is meaningless and syntactically incorrect. It is obvious that the sentence will be semantically wrong if its syntactic structures are incorrect. It can also be seen that GT does not only translate the words of the given proverb literally, but also shifts the English adjective word order into Arabic as seen in the Arabic translation. Unlike English, Arabic adjectives follow the nouns they describe. The Arabic adjectives must also agree with the nouns they describe according to decentness, gender, number and case (Al-khresheh, 2013). The word 'bites' was dealt by GT as a plural form of the noun 'bite' and was

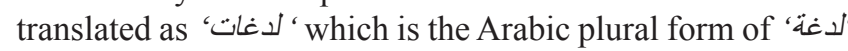
although the word 'لدغة' is inappropriate (wrong word choice) and cannot be used to describe how a dog bites. The word 'bite' has multiple meanings in Arabic and each one is limited to a certain context. The lexicon might encompass a doorway for a classical less used meaning of the plural of ' 'لدغات'. GT could not distinguish whether the word 'bites' is a verb or a noun and what the best translation is. This resulted in awkward translation. Hence, priority of general usage is reasonably required.

\section{Text 2}

English Proverb: 'Familiarity breeds contempt'

Interpretation of this proverb: Wide-ranging knowledge of someone or something causes disrespect for them or it (Ba'albaki, 2001 p, 40).

The equivalent proverb in Arabic according to Ba'albaki (2001): 'المثرة الضحك تذهب الهيبة or 'احة تذهب الئهابة' (p,40).

The second tested proverb was 'Familiarity breeds contempt' and the Arab translation was 'مفهو 'أنسال الألفة' which is completely meaningless. It resulted in an ill-formed Arabic sentence syntactically and semantically. The Arabic word ' 'أنسال' which is the translation of 'breeds' is derived from the root 'نسل 'which means 'offspring'. According to Oxford online dictionary, the word 'breed' with object means "cause (an animal) to produce offspring, especially in a controlled and organized way" (Oxforddictionaries.com, 2018). The second meaning is that "cause (something) to happen or occur, typically over a period of time" (Oxforddictionaries. com, 2018). A similar example of the given proverb is 'success breeds confidence'. Besides, the Arabic word 'الألفة' which means 'affinity' was the output of the English word 'contempt' which means 'the feeling that a person or a thing is beneath consideration, worthless, or deserving scorn." (Oxford Dictionary). In Arabic, the meaning of 'contempt' is the opposite of 'affinity'. Given this, it could be concluded that GT referred to the first meaning of the word 'breeds' instead for the second one which is the most suitable one and used the meaning of word 'affinity' as an output of the word 'contempt'.

\section{Text 3}

English Proverb: 'Charity begins at home'

Interpretation of this proverb: One's primary responsibility is for the needs of family or people close to you (Ba'albaki, $2001 \mathrm{p}, 24)$.

The equivalent proverb in Arabic according to Jabak (2008): 'الأقربون أولى بالمعروف (ph, 21).

This proverb 'Charity begins at home' gave the Arabic translation as ' through GT which is totally not the right translation of the given proverb. In comparison with the previous proverb, it could be noticed that GT uses the basic Arabic word order (VSO) in translating this proverb, beginning the translation with the Arabic word 'تبدأ which means 'start' or 'begin'. However, this is not even helpful to determine what the real meaning is. The translation of this proverb is far away from even providing a gist of the original text. The problem seems to be lexical as some English words have multiple meanings in Arabic. Specifically, GT might be misled by the form of the input because the lexicon is not well-updated to contain more suitable connotations.

\section{Text 4}

English Proverb: 'Once bitten, twice shy'

Interpretation of this proverb: This proverb means an unlikable experience encourages caution (Speake, 2007, p. 378).

The equivalent proverb in Arabic according to Jabak

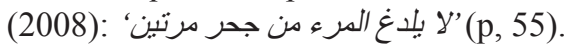

The English proverb 'Once bitten, twice shy' was tested in GT for Arabic translation and the output was 'مرة واحدة 'للعض جحر مرتين meaningless, and syntactically erroneous. This is attributed to the fact that some keywords are translated inaccurately, and for this reason they seem out of the TL context. Yet, the translation is far away from giving an accurate translation of the original text. As seen in this example, GT treats words individually, one after another neglecting the issue of word order which can incontrovertibly result in meaningless and awkward translations. A wrong analysis of the input can provide a misleading translation. For example, the word 'shy' was mistranslated as 'جر ' which means in Arabic 'hole'.

\section{Text 5}

English Proverb: 'Blood is thicker than water'

Interpretation of this proverb: Relationships within a family are the most important ones (Speake, 2007, p. 63).

The equivalent proverb in Arabic according to Jabak (2008): ' ' الدم لا يصبر ماء (p, 20). 
The researchers tested the proverb 'Blood is thicker than water' and the Arabic translation was 'الدماء اكثر كثافة من الماء 'Blin'. The same meaning can be literally obtained, but metaphorically, it is far away. However, the Arabic translation of this proverb seems to be a bit accepted syntactically as the word order is correct; conversely, the real meaning cannot be well-understood due to word choice by GT and unfamiliarity with culturally bound expressions. This highly affirms the fact of human intervention. Again, the system is misled by the form of the input. This example poses another new significant problem in the field of translation which is the issue of countability. For example, the Arabic word 'الدماء' (which means the strong relationship metaphorically) is a plural of 'blood' (uncountable noun). Some uncountable English nouns are countable in Arabic and vice versa. It can be observed that changing a singular input to a plural one can sometimes provide a misleading translation. This might be another new challenge for MT.

\section{Text 6}

English Proverb: 'A bad penny always turns up'

Interpretation of this proverb: This English proverb means that a person or thing which is disreputable, objectionable, or unnecessary tends to come into view, particularly at inconvenient times (Speake, 2007, p. 34).

The equivalent proverb in Arabic according to Jabak (2008): ' ' المنحوس منحوس (p,5)

The final proverb tested for Arabic translation in GT is ' $A$ bad penny always turns up'. The Arabic translation of this proverb was بيني سيبّة يتحول دائما ' which is literal and meaningless. The worthwhile observation here is that the classical Arabic does not have ' $p$ ' and ' $v$ '. The word 'penny' has been output as 'benny' as the corresponding script for ' $/ p /$ ' sound which is not available in Arabic and so the noun for 'penny' cannot even be translated and stored in the software. Another comment is that GT omitted the indefinite article ' $A$ ' from the output and the only interpretation is that Arabic does not have indefinite articles.

A general observation is that an awkward TL text can be seen when the SL text has a SVO order. A syntactic Arabic problem arises, as the system might have a tendency to copy the SL text. It was observed that in choosing a TL word, GT sometimes uses words that come last in priority, and this result in awkward translation. Instead, priority should be given the general usage of words. Many English words have numerous overlapping meanings in Arabic. It is the rule of GT to decide which one is the most suitable and helps in giving a coherent translation.

The problems that are seen in the above translation can be generally summarised as lexical and syntactic errors. According to Amina (2010) and Al-khresheh (2010 \& 2015), lexical problems arise when a word in one language does not have a corresponding word in the language in which one needs translation so that the word refers to the same things or ideas. In such a situation, the word may get substituted by another one and so the interpretation of translation presents errors. Particularly, the lexical errors are quite clear in the given examples. An English word might have multi- ple meanings in Arabic. It is supposed that translations of proverbs should be expressed by way of corresponding expression in the TL, taking into consideration the difficulty of finding equivalent expression in the culture. The translation process might be painless when a SL text is unambiguous and there is a good lexical match in the TL.

The second issue in translation is the syntactic errors which are due to the variation in the language structure. In the case of English and Arabic, this variation is more prominent as they belong to two unrelated families (Al-khresheh, 2016b; \& Miremadi,1991). In case of Arabic, for instance, the absence of pronoun 'it', lack of auxiliary verbs, lack of corresponding prepositional phrase, wrong word order, verb agreement, the different types of subjects and objects, the agreement of adjectives and nouns and many other grammatical functions that a language deals with might be one of the reasons for the errors in the output. Simply put, the differences in grammatical functions of both languages can be seen as the foremost syntactic problems of English-Arabic translation. This might present many challenges to online translation resulting in an awkward translation.

Generally, this study indicates that getting the most out of meaning is the ultimate and most important feature of MT. A high-quality MT scheme has to enterprise to go an extra step further than the gist level. Developing and filtering the needed rules can result in producing an acceptable and meaningful output with minimum post-editing. In the same vein, Izwaini (2006) confirmed the fact that "the less post-editing is required, the more successful the translation is, and the less time is spent and less work is done to produce the final translation" (p,146). It is worth mentioning that the lexical environment, represented by connotations and collocations, composes extremely significant and essential guides which help in selecting the most suitable equivalent. However, and as seen in the previous examples, translating proverbs from English into Arabic poses two different problems. The first one is the variability of meanings. Some English words have multiple meanings in Arabic which present many challenges to online translation. The second one is associated with the difficulty of generalization. This means that some words might collocate with one word as to produce different collocations of different meanings. This can be fixed in MT as to not generalize the meaning of a certain word that might collocate with different words, particularly in translating proverbs.

\section{CONCLUSION}

The study amid at investigating the linguistic obstacles that GT might face in carrying out the same translation from English proverbs into Arabic, and to confirm the importance of human intervention in solving the issue of accuracy. Although there have been some rapid developments and progress in translation technologies, it was found that the level of accuracy is still questionable. The general outcome of this study was that translating English proverbs into Arabic through MT faced many obstacles. This was evident in providing wrong TL equivalent, literal translation, wrong word order, inappropriate lexical words, and grammatical 
mistakes. Words with multiple meanings, unfamiliarity with culturally bound expressions as well as the different linguistic structures between English and Arabic might create such obstacles. Consequently, relying solely on MT to translate sentences which have ambiguity such as proverbs was a matter of complexity. Thus, human translation cannot be replaced by MT. It is totally disagreeable that MT is accepted blindly. Rather, it is always in need for a human touch.

The study suggests that if the MT is fed by a good interpretation of both English and Arabic proverbs, then a correct and acceptable online translation might be obtained. As many English proverbs do not have an equivalent in Arabic, the software can give an interpretation of the proverb as an alternative for the user to have a correct output. To have such interpretations, a human intervention is highly required. It is believed to be the best way to improve quality and accuracy of MT. This can be done by having experts from both languages who can develop and create a good database in order to produce a better quality of translation. Filtering and developing the system to decide first the target context through checking the essential words as well as the semantic field of the text can help in reducing inappropriate TL equivalents. Additionally, Lexically, proverbial items should not be translated individually. Choosing the most suitable alternative in deciding the accurate meaning cannot be without taking the whole lexical environment, connotations, and collocations into consideration. In case that there are no alike words in Arabic, the system should be updated to have different suggestions.

The paper had some limitations such as lack of a large number of proverbs as data for analysis. As there are many MTs, a comparative study in terms of accuracy is suggested for further research. There should be more research done in this field so as to get a better quality of online translation, revealing the fact that proverbs remain the most influential and powerful implementation for transmitting cultural ideas of one generation to another.

\section{REFERENCES:}

Ahmed, S. (2005). Educational and social values expressed by proverbs in two cultures: Knowledge and use of proverbs in Sudan and England. Unpublished PhD dissertation, University of Technology, Berlin.

Al-khresheh, M. (2010). Interlingual interference in the English language word order structure of Jordanian EFL learners. European Journal of Social Sciences, 16(1), 106-113.

Al-khresheh, M. (2013). The misuse of word order in the writing of jordanian EFL learners. Unpublished doctoral dissertation, University of Malaya, Kuala Lumpur, Malaysia.

Al-khresheh, M. (2015). A review study of interlanguage theory. International Journal of Applied Linguistics \& English Literature, 4 (3), 124 - 131.

Al-khresheh, M. (2016A). A review study of error analysis theory. International Journal of Humanities and Social Science Research, 2, 49-59.
Al-khresheh, M. (2016B). A review study of contrastive analysis theory. Journal of Advances in Humanities and Social Sciences, 2 (6), 330-338.

Alshammari, J. N. (2015). Examining Nida's translation theory in tendering Arabic proverbs into English: A comparative analysis study. International Journal of English Language and Linguistics Research, 3 (8), 45-57.

Amina, A. (2010). Lexical translation problems: The problem of translating phrasal verbs. The case of third year LMD learners of English. Published Master Thesis, the University of Mentouri, Algeria. Retrieved from https:// bu.umc.edu.dz/theses/anglais/AYA1194.pdf

Anastasiou, D. (2010). Idiom treatment experiments in machine translation. Published Doctoral Dissertation, Saarland University, Germany. Retrieved from https:// pdfs.semanticscholar.org/b263/a3a62f65df780e95bc86fa43fd9ce38e5186.pdf

Ba'albaki, M. (2001). A modern English-Arabic dictionary. Dar El-ilm LiL MALAYEN: Lebanon.

Bassnett, S. (2007). Culture and translation. In. P. Kuhiwczak, and K. Littau (Eds.), A companion to translation studies, 13-23. Clevedon, Avon, UK: Multilingual Matters.

Boitet, C., Hervé B., Mark S., \& Valérie B. (2009). Evolution of MT with the Web. In Proceedings of the Conference Machine Translation 25 Years On, 1-13. Cranfield: Bedfordshire.

Breed. (2018). oxforddictionaries.com. Retrieved from https://en.oxforddictionaries.com/definition/breed

Brown, P., J, Cocke, S. Della Pietra, F. Jelink, V. Della Pietra, J. Lafferty, R. Mercer and P. Rossin. (1990). A statistical approach to machine translation. Computational Linguistics, 16, 79-85.

Carpaut, M. (2013). A semantic evaluation of machine translation lexical choice. In Proceedings of the $7^{\text {th }}$ Workshop on Syntax, Semantics and Structure in Statistical Translation, 1-10. Retrieved from http://www.anthology.aclweb.org/W/W13/W13-08.pdf\#page=11

Dabaghi, A., Pishbin, E., Niknasab, L. (2010). Proverbs from the viewpoint of translation. Journal of Language Teaching and Research, 1 (6), 807-814.

Denkowski, M. (2015). Machine translation for human translators. Unpublished doctoral dissertation, Carnegie Mellon University, Pittsburgh, Pennsylvania.

Dorr, B, J. (1994). Machine translation divergences: A formal description and proposed solution. Computational Linguistics, 20 (4), 597-633.

Feng, S., Kang, S,J., Kuznetsova, P., \& Choi, Y. (2013). Connotation lexicon: A dash of sentiment beneath the surface meaning. In Proceedings of the $51^{\text {st }}$ Annual Meeting of the Association for Computational Linguistics, Association for Computational Linguistics, Sofia, Bulgaria.

Ghazala, H. (1995). Translation as problems and solutions: A course book for university students and trainee translators. Beirut: Dar wa Maktabat Al-Hilal.

Hannouna, H, Y. (2004). Evaluation of machine translation systems: The translation quality of three Arabic systems. Unpublished doctoral dissertation, Al-Mustansiriyya University, Baghdad,Iraq. 
Hatim, B., \& Munday, J. (2004). Translation: An advanced resource book. Oxford: Routledge.

Honeck, R. (1997). A proverb in mind: the cognitive science of proverbial wit and wisdom. USA: Lawrence Erlbaum.

Ilyas, I, A. (2013). The importance of connotation in literary translation. Arab World English Journal, (1), 248-263.

Izwaini, S. (2006): "Problems of Arabic machine translation: Evaluation of three systems." Proceedings of the international conference "The challenge of Arabic for NLP/MT", The British computer society (BSC), London, 118-148.

Jabak, O, O. (2008). One thousand and one English proverbs translated into Arabic (1 ${ }^{\text {st }}$ ed.). (n.p): Author.

Jacobson, S. (1968). The problem of describing syntactic complexity. Studia Neophilologica, 40(1), 114-129.

Khalifa, M, E. (2015). Problems in translating English and Arabic languages' structure: A case study of EFL Saudi students in Shaqra university. European Journal of English Language and Literature Studies. 3 (4), 22-34.

Madsen, M. W. (2009). The limits of machine translation. Published Master Thesis, Copenhagen University,Copenhagen, Denmark. Retrieved from http://vantage-siam.com/upload/casestudies/file/file-139694565.pdf

Meider, W. Dundes, A. (1994). The wisdom of many: Essays on the proverb. Wisconsin: University of Wisconsin Press.

Mieder, W.(2004). Proverbs: A handbook. London: Greenwood Press.

Mollanazar, H. (2001). Principles and methodology of translation. Tehran: SAMT.

Newmark, P. (1988). A textbook of translation. New York: Prentice hall.

Nida, E. A. (1964). Toward a science of translating: with special reference to principles and procedures involved in Bible translating. Brill Archive.
Resnik, P., \& Yarowsky, D. (1999). Distinguishing systems and distinguishing senses: New evaluation methods for word sense disambiguation. Natural Language Engineering, 5(2), 113-133.

Thalji, B, M. (2015). The translation of proverbs: Obstacles and strategies. Published Master Thesis, Middle East University, Amman, Jordan. Retrieved from https://meu. edu.jo/libraryTheses/5871fd344b5b6_1.pdf

Sameer, H, I. (2016). A cognitive study of certain Animals in English and Arabic Proverbs: A Comparative Study. International Journal of Language and Linguistics, 3 (5), 133-143.

Samovar, L., Porter, R., \& McDaniel, E. (2009). Communication between cultures ( $7^{\text {th }}$ ed., 198-214). Stamford, CT: Cengage Learning.

Sidhu,K, B., Singh, A., \& Goyal, V. (2010). Identification of proverbs in Hindi text corpus and their translation into Punjabi. Journal of Computer Science and Engineering, 2 (1), 32-37.

Speake, J.(2007). The Oxford dictionary of proverbs ( $5^{\text {th }}$ ed.).Oxford, United Kingdom: Oxford University Press.

Syzdykov, K. (2013). Contrastive studies on proverbs. Procedia - Social and Behavioral Sciences, 136 (2014), 318-321.

Taravella, A., \& Villeneuve, A. O. (2013). Acknowledging the needs of computer-assisted translation tools users: the human perspective in human-machine translation. The Journal of Specialized Translation, 19(January), 62-74.

Zheng1, H. (2015). A case study of machine translation: Problems and suggestions. International Journal of English Linguistics, 5 (2): 92 - 99. 


\section{APPENDICES/SAMPLES}

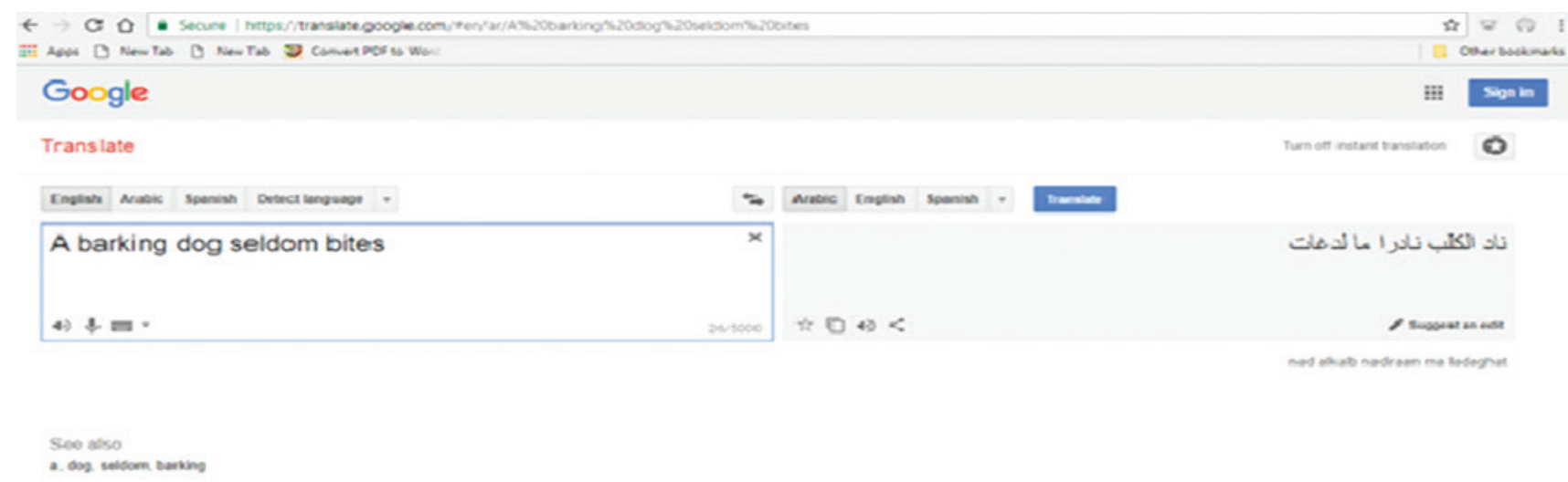

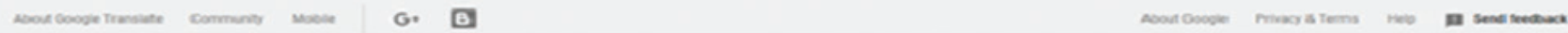

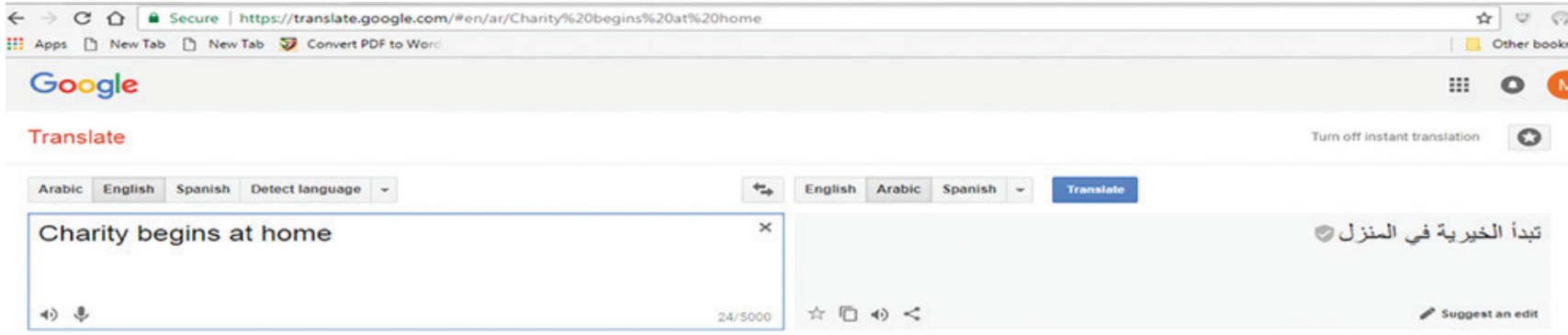

See also
charity bogins at home, home, at, at home

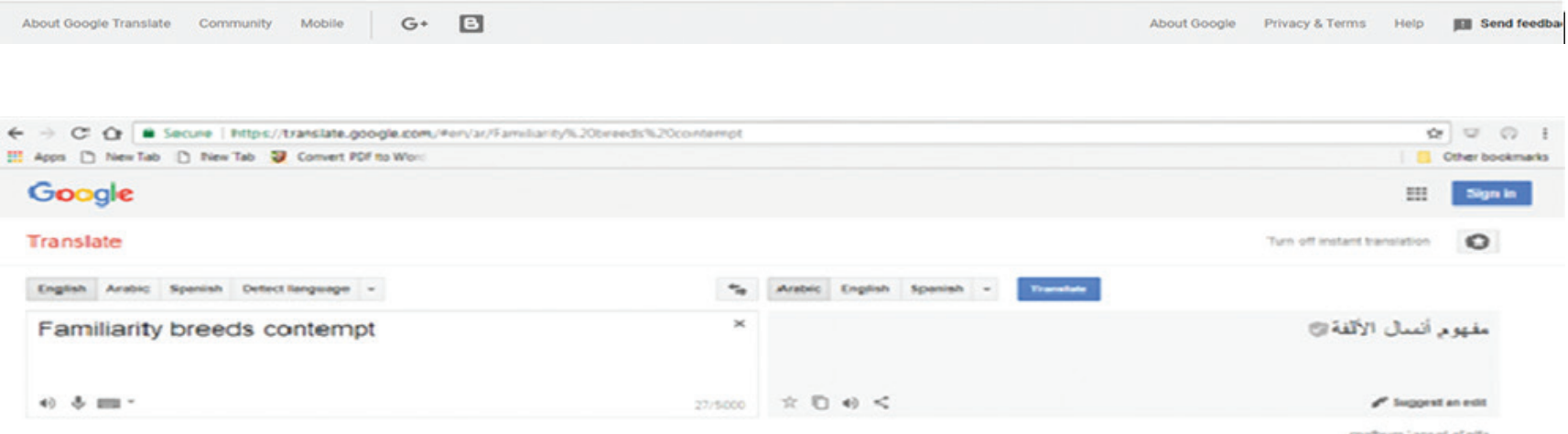

Seen orso

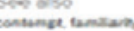




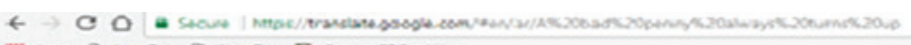

$\therefore$ ० $\odot$ :

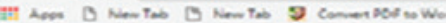

ili

Google

○

Translate

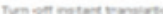

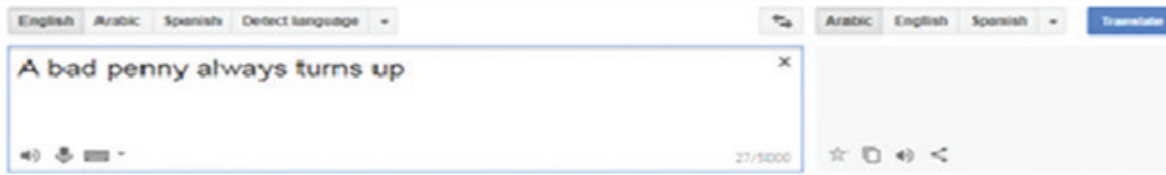

A بئش سينة يتحورل دانسا A

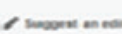

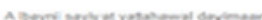

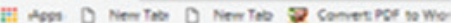

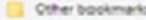

\section{Google}

Translate

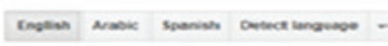

Once bitten, twice shy

4) $4=$

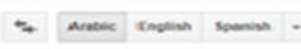

(2) 5000 से 0 \% $<$

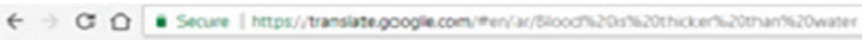

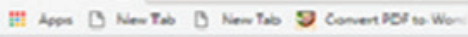

Google

Translate

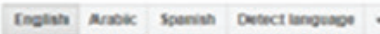

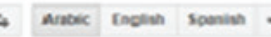

Blood is thicker than water

क)
क $\nabla \leqslant$ :

\section{iff $\sin$ in}

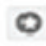

See also

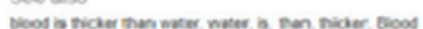

